\title{
A SURGICAL STUDY OF COARCTATION OF THE AORTA
}

\author{
BY \\ ROWAN NICKS \\ From the Cardio-Thoracic Surgical Unit, Green Lane Hospital, Auckland, New Zealand* \\ Received June 21, 1957
}

The object of this study is to present our experience in dealing with coarctation of the aorta and to discuss policy in the management of individual patients who have widely varying ages and symptoms, sometimes complicated by additional lesions. Surgical treatment, which is on a national basis, was developed rather hesitantly at first, but is now practised with confidence. Forty-four cases have been seen since 1948 of whom twenty-seven have received surgical treatment.

\section{Analysis of Necropsy Data for New Zealand}

The number of deaths associated with coarctation of the aorta registered in New Zealand and confirmed by necropsy over the past nine years is 28 , an average of three cases a year. These have been analysed in Table I. In this series 46 per cent of the deaths occurred in the first year of life and, if these cases are excluded, the average survival was 29 years, 70 per cent of the deaths occurring between 30 and 59 years of age.

TABLE I

ANalysis of Deaths from Coarctation in New Zealand, 1947-1955

\begin{tabular}{|c|c|c|c|c|}
\hline \multirow{2}{*}{$\begin{array}{l}\text { Age } \\
\text { groups }\end{array}$} & \multicolumn{2}{|c|}{ Sex } & \multirow{2}{*}{ Total } & \multirow{2}{*}{ Cause of death } \\
\hline & $\mathbf{M}$ & $\mathbf{F}$ & & \\
\hline $\begin{array}{l}0-1 \\
2-10 \\
11-20\end{array}$ & $\begin{array}{l}7 \\
1 \\
3\end{array}$ & $\begin{array}{l}6 \\
1 \\
1\end{array}$ & $\begin{array}{r}13 \\
2 \\
4\end{array}$ & $\begin{array}{l}\text { Broncho-penumonia in } 6 \text { and C.H.F. in } 7 \\
\text { Endarteritis in } 1 \text { and post-operative in } 1 \\
\text { Ventricular fibrillation in 2, C.H.F. in 1, and } \\
\text { operation in } 1\end{array}$ \\
\hline $\begin{array}{l}21-30 \\
31-40\end{array}$ & $\overline{2}$ & $\overline{2}$ & $\overline{4}$ & $\begin{array}{l}\text { C.H.F. in } 2 \text {, rupture in } 1 \text {, and coronary insuffi- } \\
\text { ciency in } 1\end{array}$ \\
\hline $41-60$ & 5 & - & 5 & C.H.F. in 4 and rupture in 1 \\
\hline Totals $\ldots$ & 18 & 10 & 28 & \\
\hline
\end{tabular}

C.H.F. $=$ Congestive heart failure.

The true incidence in this necropsy series is impossible to assess accurately as no detailed information as to the number of necropsies performed in New Zealand over the period of study is available, but it is at least one in 3000 deaths, compared with one in 1000 (Blackford, 1927), and one in 1550 (Calodney and Carsen, 1950). The predominance of male to female subjects in both series is in accordance with accepted figures.

* Delivered to the Australasian Cardiac Society, Wellington, March 2, 1956. Author's address: Royal Prince Alfred Hospital, Sydney, Australia. 


\section{Clinical Series}

We have seen 44 patients, 14 in the first decade, 13 in the second, 10 in the third, 4 in the fourth, 2 in the fifth, and 1 in the sixth decade. This means 3 per 100,000 for the Province of Auckland and rather under 2 per 100,000 for the remainder of North Island and for South Island, but there are no doubt other cases that have not been seen. All our 44 patients were European and no Maoris were seen. The infrequency of recognized coarctation in coloured races has been commented upon by King (1926).

Clinical Presentation. The symptoms have been minor and related to vascular hypertension: on the average, this has not been excessive before fifteen years (about 140 systolic), has risen to a maximum between twenty and thirty years (200/105), and fallen slightly after this. The pressure in individual cases, however, differed little when taken years apart, suggesting that the heart was capable of functioning normally against the resistance until breakdown from congestive heart failure, cerebro-aortic vascular accidents, or endocarditis.

Usually the correct diagnosis was inferred by observing vigorous pulsation in the neck and recognizing delay or absence of the femoral pulses, attention having been drawn to the possibility of coarctation by brachial hypertension or by unexplained systolic murmurs. It was confirmed by clinical evidence of collateral anastomotic vessels, fluoroscopic demonstration of lesser degrees of left ventricular hypertrophy with evidence of aortic constriction indicated by a small or double aortic knuckle, dilatation of the ascending aorta, and notching of the lower ribs from tortuous intercostal arteries, all of these signs being more evident in older cases with more severe degrees of coarctation. The gravity of the obstruction in individual cases has been estimated by the blood pressure, and the degree of cardiac hypertrophy as recorded by serial X-rays and electrocardiograms. Blood pressures have been taken from both brachial arteries to ascertain the site of the lesion in relation to the aortic arch and its branches. In most cases with left ventricular hypertrophy there has been a rounding and elongation of the left ventricle without significant enlargement in the transverse diameter of the heart, the cardio-thoracic index being within normal limits in 33 of the 44 cases, doubtfully enlarged in 4 , and moderately increased in 7 cases, three of which were complicated by associated cardiac lesions.

Angiocardiography is regarded more as an interesting record than of great practical value except in unusual cases, and when performed, has merely confirmed visually the presence of a lesion already deduced. The operability in each case has been assessed at thoracotomy. The routine provision of freeze-dried arterial grafts has lessened still further the scope of this examination.

\section{Associated Lesions in the Clinical Series}

A ventricular septal defect was considered probable in two patients. In one, a child of 8 years, death occurred but no necropsy was available. In the other, a man of 36 , the clinical condition has remained satisfactory for three years.

Aortic valve lesions have been found in 7 cases (16\%). In six the presence of a bicuspid aortic valve was suspected: in four it has produced no effect on the dynamics although in two of them there was slight aortic regurgitation which did not interfere with a satisfactory operative result, but in the other two there was serious regurgitation (one complicated by angina pectoris as well), and great cardiac enlargement, so that surgery was not advised. In a girl, aged 16, who had enough aortic regurgitation to produce a blood pressure of 200/70 before operation, the resection was successfully carried out and a year later the aortic regurgitation seemed less as the blood pressure was $155 / 95$. In a man, aged 21 , however, with a blood pressure of $165 / 60$, the regurgitation was thought too great to advise operation: four years later, although he was still free from symptoms, the heart had become much larger.

A diagnosis of subaortic stenosis was made in a child of eight without symptoms and operation has been deferred until she is older.

Turner's syndrome has occurred in one woman and two adolescents, all of whom have received operative treatment. 
Pulmonary emphysema was present in two cases. Resection of the coarctation was undertaken in one of these, a man, aged 21, who also had pulmonary hypertension, 74/40 mm. Hg. He had a stormy convalescence with some congestive heart failure, but improved gradually. A year later he was better with a blood pressure of $150 / 80$ instead of $230 / 115$, but still with right bundle-branch block.

\section{SURGICAL TREATMENT}

At first the policy was conservative and operation was deferred while the clinical status was satisfactory. After successful relief by operation, even in cases with severe cardiovascular embarrassment, it is now advised in most cases at the appropriate time, and we think this is generally accepted. This policy is illustrated by the histories of two of our patients, one of whom was made sterile to prevent the strain on her coarctation of further pregnancy, and the other rendered impotent by bilateral lumbar sympathectomy (performed elsewhere) on the mistaken diagnosis of essential hypertension.

In the twenty-seven cases between the ages of 9 and 35 years in which surgery has been done, resection has been performed in all. The aorta was reconstituted by end-to-end anastomisis in twenty-four, and by a plastic reconstruction in two. In one patient hæmorrhage from a friable suture line necessitated terminal obliteration of both ends of the aorta, and this patient has survived without much change for over two years. Two cases died from operation (mortality $7 \%$ ) one from haemorrhage and one from cerebral anoxia. There have been no late deaths.

Complications of Operation. Severe hæmorrhage occurred in three cases apart from the two already described, one from cutting through of the proximal toothed Pott's clamp on the aorta at the medial end (controlled with difficulty), and one each from rupture of an aortic intercostal and a bronchial vessel respectively.

Post-operative chylothorax developed immediately in one cases (necessitating suture of the thoracic duct), temporary recurrent laryngeal palsy in one, and transient pulmonary collapse in two others. One of these last two was complicated by pulmonary hypertension and emphysema, and the collapse and infection induced early cardiac-respiratory failure from which there was a prolonged and stormy convalescence.

Blood Pressure Changes. The blood pressure has been recorded in most cases during and after operation. On application of the clamps above and below the coarctation there was an immediate rise to a high stationary level in the proximal segment if the subclavian artery had not been occluded; but often it continued upwards insidiously during the operation if this vessel were occluded. The hypertension was controlled by raising the trunk or by the administration of an intravenous normal saline drip containing an $\mathbf{0 . 1}$ per cent solution of the hypotensive drug arfonad during the period of occlusion. In spite of careful regulation, on occasions the blood pressure was observed to fall precipitately with the exhibition of this drug, but no harm ensued from this.

In the distal segment there was a regular fall in the systolic and diastolic pressure, but a good flow was observed. On release of the clamps after resection and restoration of the aorta there was an immediate fall in both systolic and diastolic blood pressures with equalization across the suture line. In some cases, to maintain and stabilize the blood pressure at the arbitrary systolic level of $100 \mathrm{~mm}$. $\mathrm{Hg}$, it has been useful to lower the upper half of the body and to speed up the blood transfusion for a short period. In one case, already mentioned, hypotension on release of the aortic clamps was considered to have caused irreversible brain damage.

As an illustration, a case aged 10, may be quoted. Before clamping the aorta at operation, the blood pressure was $141 / 99$ proximally and 123/92 distally; during the clamping the difference increased, the figures being $160 / 111$ and $78 / 74$, and after release of this there was a balanced pressure of $116 / 87 \mathrm{~mm}$. $\mathrm{Hg}$.

Post-operatively the patients have been in a state of vaso-constriction with a variable rise of blood pressure and pulse rate, which has relaxed only gradually. The blood pressure has remained high, sometimes at the same level as before operation, for one to three weeks and has usually not 
attained a normal level at the time of discharge. At the follow-up examination (minimum four months), the blood pressure has been reduced to normal in 19, reduced but not to normal in 4 , and unchanged in one case in which anastomosis was not accomplished. The temporary persistence of hypertension after restoration of the aortic lumen is thought to be due to vaso-constrictive reflexes attempting to maintain the habitual arterial pressure. Post-operative rupture at the suture line is considered unlikely unless there has been either a technical fault or infection, and our cases have been allowed to walk about as soon as the blood pressure and pulse rate were stabilized.

Results. Of the 29 patients who have had operations, two died and details have been given. None is known to have died since the operation so far, but we have not been able to trace three of them. Nineteen have obtained an excellent result, by which we mean that each is leading a normal life with a normal blood pressure and a normal electrocardiogram. Four others still have some minor restrictions and a small rise of blood pressure, so we have called this a good result instead of excellent. Only one has failed to obtain any improvement.

\section{The Local Pathological Lesion}

The coarctation is seen at operation as a localized constriction of the anterior, posterior, and superior aspects of the aortic isthmus adjacent to the insertion of the ligamentum arteriosum. It has been remarkably uniform, differing only from this standard pattern in four cases, one with hypoplasia of the arch, one with a partial constriction extending into the transverse aorta proximal to the subclavian artery, and two with the coarctation just at the origin of this artery (see later). In the adult cases operative resection and anastomosis has been facilitated by the natural lengthening of the vessels in response to hypertension. Examination of the excised specimens in each case has disclosed a diaphragm with a perforation, usually eccentric, that constitutes the sole aortic lumen and is situated at the site of the maximum external narrowing. Distal to this, a jet lesion, described by Edwards (1948) with thickening and irregularity of the intimal surface and with underlying atheroma has been observed. This is important since it is prone to saccular and dissecting aneurysms and is potentially dangerous for suture.

The lumen has been absent in some cases and has varied from a pin-hole up to $4 \mathrm{~mm}$. in diameter in the others. This maximum diameter, found in a case with severe hypertensive symptoms, might represent a critical size that produces enough obstruction to give symptoms. Microscopic sections confirmed a constant thickening of the tunica media which was infiltrated by varying amounts of fibro-elastic tissue and smooth muscle. This actually formed the coarctation diaphragm except for intimal thickening of fibrous tissue at the free margin, indicating that there would be no local weak-
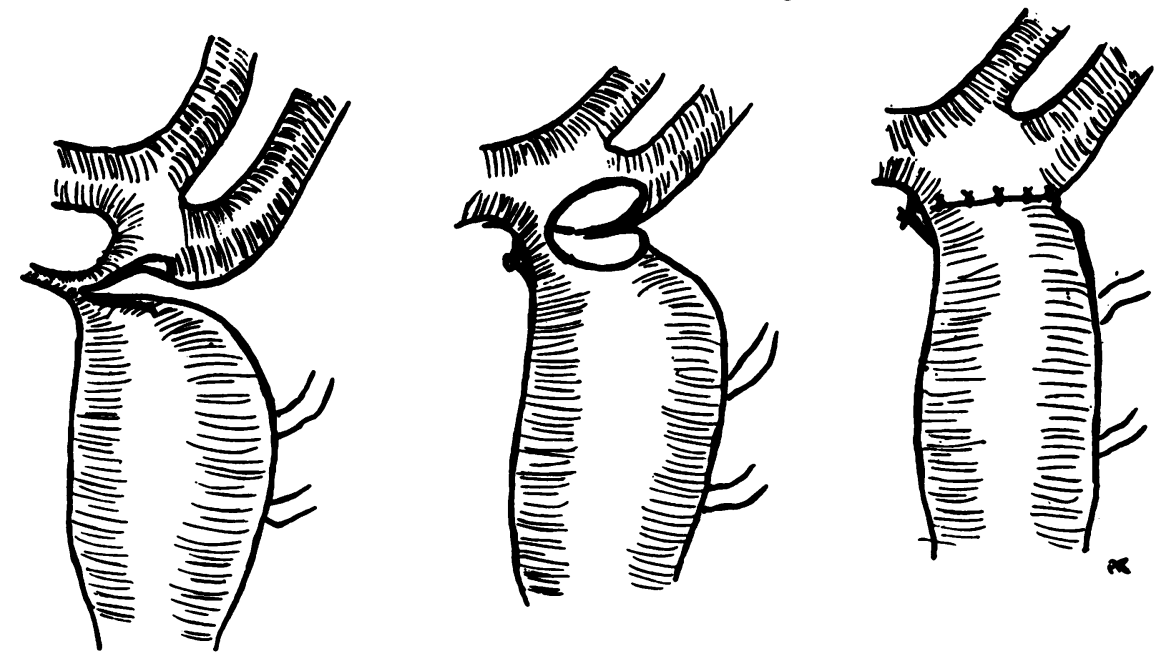

FIG. 1.-Plastic operation for high coarctation (see p. 355). 
ness of the aorta if the diaphragm were judiciously excised. The ductus has been patent in only one case: it was small and closely proximal to the coarctation.

These were the usual findings but sometimes, as stated, the coarctation was unusual in site or shape. A man, aged 33, had severe headaches and a blood pressure of 190/110. At operation the coarctation was very high, short, and situated just beyond the subclavian artery, with an almost aneurysmal post-stenotic dilatation beyond (Fig. 1). The subclavian artery and the fusiform vessel lay closely together. A plastic operation excising the coarctation diaphragm from within and restoring the lumen between the subclavian and distal aorta was performed, and his convalescence was uneventful.

In another operation on a boy, aged 13, who had no symptoms and only moderate hypertension, the coarctation was at the origin of the left subclavian artery and extended upwards into the transverse aorta. Surgical relief was accomplished by a plastic operation (Fig. 2), during which the pressures in the aorta were recorded as $180 / 135$ above the clamps and 94/87 below, falling to $130 / 98$ above and 120/97 below immediately after the operation. The blood pressure reading later was $140 / 95$ and the final result at follow-up was excellent.
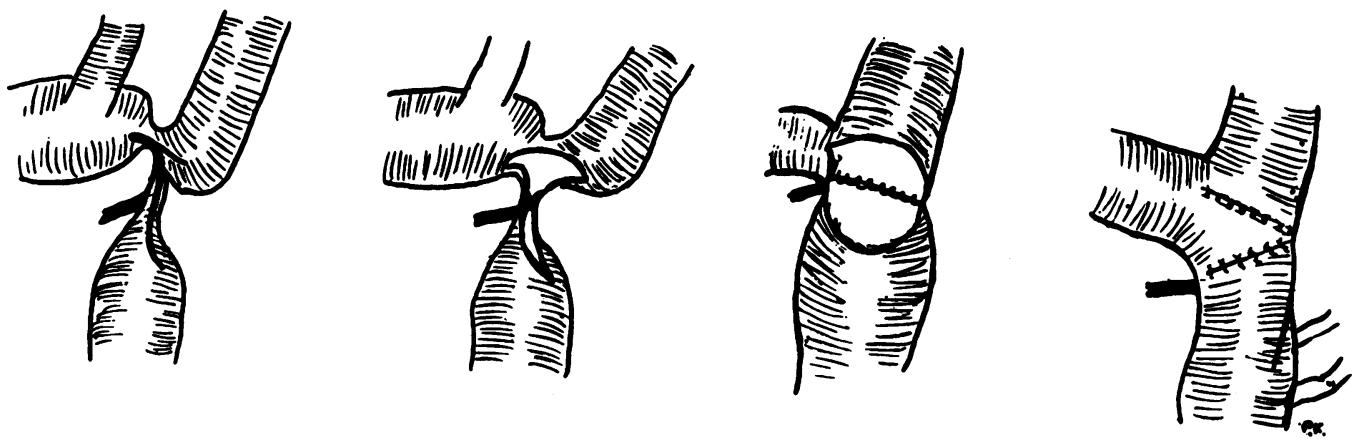

FIG. 2.-Diagrammatic representation of a plastic operation for coarctation extending into the transverse aorta (see text).

\section{Discussion}

The single case referred during infancy is noteworthy in view of the deaths recorded during the first year of life (Table I) and of a study of Ober and Moore (1955) who found that ten per cent of deaths from congenital heart disease were from this cause. Mustard et al. (1955) in a report on cases of coarctation coming to necropsy at the Children's Hospital, Toronto, found preductal infantile coarctation to be fatal in 89 per cent of cases within three months and the adult type within six months in 60 per cent of their cases. The period of infancy is generally accepted as less critical in localized constrictions of the adult type, as the heart is not subjected to an intolerable strain without a by-pass of collaterals as it is in the atretic group.

In view of these figures, recognition of coarctation in infancy is a challenge so that surgical relief may be considered, despite the risk, in cases of progressive heart failure. The danger of spinal cord damage and of operative shock may be lessened by conducting the operation under hypothermia (Gerbode et al., 1955) at which time the natural elasticity of the tissues permits resection of considerable lengths of atresia and reconstitution of the open ends.

The need for surgical correction (Crafoord and Nylin, 1945) is now more generally accepted. The average predictable life for adults with coarctation, based on post-mortem studies, is between 30 and 35 (Reifenstein et al., 1947; Lewis, 1933), death usually taking place from cardiac failure, vascular accidents, or infective endocarditis and endarteritis. The hazard of a 60 per cent (Claggett et al., 1955) or 74 per cent (Abbott, 1936) chance of death before forty years, even in symptomless cases, seem to justify even the present surgical mortality of 7 per cent from this operation which 
has been without serious post-operative complications in most hands and has restored the cardiovascular system towards normal in most cases. Attention has been drawn to the risks of pregnancy in association with coarctation (Mendelson, 1940; Rosenthal, 1955), and to the special danger of rupture in association with a bicuspid aortic valve (Abbott, 1936).

The surgical mortality is reported to be less and the operation has been found technically easier in the young, where the tissues are resilient and the intercostal vessels smaller. Now that the accumulation of cases has been cleared it has been necessary to postulate an age of elective operation to anticipate future complications, and this has arbitrarily been chosen at about fourteen years, before hypertension is severe and dangerous, and when the aorta is of such a size that the obstruction will not be significant even if the aorta does not grow at the suture line. Operation has not been withheld at any age if there is impending cardiac or vascular damage. In this series surgery has been deferred in children and in adults with only a moderate rise of blood pressure and no evidence of cardiac strain. It was withheld in two cases (already mentioned) in which severe aortic regurgitation was considered the dominant lesion.

There has been no undue hazard in older cases, even though the vessels and the collaterals were larger; and, indeed, elongation of the vessels resulting from prolonged hypertension has usually enabled approximation with less difficulty. Arterial grafts, although available, have not been necessary, approximation of the ends having been possible in all but one case. Use would be made of a graft if required after excision of a long atretic segment, local vascular damage, or an aneurysm, as recommended by Claggett $e t$ al. (1955), who reported successful insertion in 4 of 177 cases.

The development of an operation for local excision of the diaphragmatic obstruction and reconstruction of the aorta by local plastic procedures may lower the mortality and give better results in suitable cases, especially in high coarctations involving the aortic arch (Fig. 1 and 2).

\section{SUMMARY}

A brief clinical study of 44 consecutive cases of coarctation is presented, together with an analysis of deaths occurring in New Zealand over the past nine years. The beginning of this period coincides with the formation of the Cardiosurgical Unit.

The local pathological malformation is discussed on the basis of operation specimens.

The surgical treatment and follow-up results of 29 cases submitted to surgery are reported. The blood pressure changes during and after operation are described.

Coarctation in infancy is briefly considered and a plea raised for the early diagnosis and surgical treatment of this condition in cases suffering from progressive heart failure. An arbitrary age of fourteen years is put forward as the time of election for routine surgery, to be varied with individual circumstances, and evidence of cardiovascular breakdown. A local plastic operation is recommended for high coarctation in the region of the aortic arch.

The author wishes to thank the members of the Cardiosurgical Unit for permission to discuss these cases. The operations were performed by Mr. Douglas Robb or the author. The follow-up was facilitated by help from Mr. D. Cole, Dr. P. Molloy, and Miss P. Kerkin.

\section{REFERENCES}

Abbott, M. E. (1936). Atlas of Congenital Heart Disease. American Heart Association.

Blackford, L. M. (1927). Collect. Papers Mayo Clin., 19, 686.

Calodney, M. M., and Carson, M. (1950). J. Pediat., 37, 46.

Clagett, O. T., Kirklin, J. W., and Ellis, F. H. (1955). Surg. Clin. N. Amer., 35, 937.

Crafoord, C., and Nylin, G. (1945). J. thorac. Surg., 14, 347.

Edwards, J. E. (1948). Proc. Staff. Med. Mayo Clin., 23, 108.

Gerbode, F., Purdy, A., Alway, R. H., Piel, J. J., and Da Costa, I. A. (1955). Amer. J. Surg., 89, 1138.

King, J. T. (1926). Arch. intern. Med., 38, 69.

Lewis, T. (1933). Heart, 16, 205.

Mendelson, C. L. (1940). Amer. J. Obstet. Gynec., 39, 1014.

Mustard, W. T., Rowe, R. D., Keith, J. D., and Sirek, A. (1955). Ann. Surg., 141, 429.

Ober, W. B., and Moore, T. E., Jr. (1955). New Zealand J. Med., 253, 271.

Reifenstein, G. H., Levine, S. A., and Cross, R. E. (1947). Amer. Heart J., 33, 146.

Rosenthal, L. (1955). Brit. med. J., 1, 16.

Tubbs, O. S. (1955). Brit. med. Bull., 11, 197. 